\title{
Prevalência de obesidade geral e central na população adulta na cidade de Tubarão, Santa Catarina: um estudo de base populacional
}

\author{
Prevalence of obesity among adults in Tubarao city, Southern Brazil: \\ a population-based study
}

\author{
Mariana Soares Pereira' ${ }^{1}$ João Carlos Fantini Schaefer ${ }^{1}$, Marina Constante Dutra ${ }^{1}$, Estevão José Muller Uliano ${ }^{1}$, \\ Clovisa Rech de Jesus", Brian de Carvalho Silvestre', Danúbia Felippe Grassi de Paula Machado ${ }^{3}$, Daisson José Trevisol ${ }^{2,3}$, \\ Fabiana Schuelter Trevisol ${ }^{2,3}$
}

'Estudantes de Graduação em Medicina da Universidade do Sul de Santa Catarina (UNISUL), Tubarão, Santa Catarina; ${ }^{2}$ Doutores em Ciências da Saúde/Cardiologia pela Universidade Federal do Rio Grande do Sul, Professores do Programa de Pós-Graduação em Ciências da Saúde e do Curso de Graduação em Medicina da UNISUL, Tubarão, Santa Catarina; ${ }^{3}$ Centro de Pesquisas Clínicas do Hospital Nossa Senhora da Conceição, Tubarão, Santa Catarina.

\section{RESUMO}

Objetivos: Estimar a prevalência de obesidade geral, obesidade central e fatores associados em adultos de uma cidade na região Sul do Brasil. Métodos: Estudo transversal de base populacional com adultos residentes em Tubarão, Santa Catarina, de novembro de 2011 a junho de 2012. Os participantes foram entrevistados e submetidos à antropometria. A entrevista avaliou dados sociodemográficos, atividade física, consumo de álcool, tabagismo, hábitos alimentares (passos da alimentação saudável) e dados clínicos. A associação entre as variáveis de interesse foi avaliada pelo teste de qui-quadrado de Pearson, e para comparação entre as médias utilizou-se o teste t de Student. Empregou-se o teste de Kappa para verificar o grau de concordância entre os dois tipos de obesidade. Para controle dos fatores de confusão, foram incluídas na análise as variáveis que apresentaram valor de $\mathrm{p}$ abaixo de 0,20 e/ou selecionadas com base na revisão de literatura, sendo feita análise multivariada pela regressão de Poisson.

Resultados: Foram estudados 371 adultos, com média de idade de 40,5 ( $\pm 12,3$ anos), sendo que $237(63,9 \%)$ eram mulheres. A obesidade geral (aferida pelo índice de massa corporal) em homens e mulheres foi de $23,1 \%$ e $29,1 \%$, respectivamente (diferença não significativa). A obesidade central (medida pela circunferência abdominal) foi verificada em $24,6 \%$ dos homens e 43,5\% das mulheres ( $p<0,001$ ). Ao se verificar o grau de concordância entre a obesidade geral e central obteve-se um valor de Kappa de 0,7 (bom grau de concordância). Os participantes não obesos apresentaram uma média de 4,3 passos da alimentação saudável contra 3,7 dos participantes com obesidade central ( $\mathrm{p}<0,001)$ e $3,7 \mathrm{com}$ obesidade geral $(\mathrm{p}=0,002)$. Com relação à obesidade geral, houve uma associação independente com faixa etária maior, histórico familiar de obesidade e uso de moderadores do apetite. Com relação à obesidade central, houve associação independente com sexo feminino, maior faixa etária, ter união estável, história familiar de obesidade e uso de moderadores do apetite.

Conclusões: A prevalência de obesidade geral foi de $26,9 \%$, e os fatores associados foram idade mais avançada e predisposição genética. A prevalência de obesidade central foi de $36,7 \%$, e os fatores associados foram sexo feminino, idade mais avançada e união estável. Houve boa concordância entre obesidade central e geral. A dieta saudável foi fator de proteção para obesidade geral e central. O uso de medicamentos para emagrecer esteve associado aos dois tipos de obesidade.

DESCRITORES: ADULTO; OBESIDADE; OBESIDADE ABDOMINAL; ÍNDICE DE MASSA CORPORAL; CIRCUNFERÊNCIA ABDOMINAL; FATORES DE RISCO.

\section{ABSTRACT}

Aims: To estimate the prevalence of overall and central obesity, and associated factors in adults in southern Brazil.

Methods: A cross-sectional, population-based study was conducted among adults living in Tubarao, State of Santa Catarina, Brazil, between November 2011 and June 2012. Participants were interviewed, and anthropometric measurements were taken. The interview assessed sociodemographic data, levels of physical activity, alcohol consumption, smoking, dietary habits and clinical data. The association between the variables of interest was tested using Pearson's Chi-square; Student t-test was used to compare means, and agreement between both types of obesity was assessed using Kappa statistics. Modified Poisson's regression analysis was performed including variables with $\mathrm{p}<0.2$ as well as variables selected through literature review.

Results: We studied 371 adult patients (mean age $40.5 \pm 12.3$ years; $63.9 \%$ women). Overall obesity prevalence was $23.1 \%$ for men and $29.1 \%$ for women (no statistical difference between them). Waist circumference measurement revealed that $24.6 \%$ of men and $43.5 \%$ of women had central obesity. Kappa value for the agreement between central and overall obesity was 0.7 (good level of agreement). Non-obese participants showed an average of 4.3 healthy feeding steps versus 3.7 steps of subjects with central and overall obesity (respectively $p<0.001$ and $p<0.002$ ). Regarding overall obesity, there was an independent association with younger age, family history of obesity and use of diet pills. Regarding central obesity, there was an independent association with female gender, older age, stable marital relationship, family history of obesity, and use of diet pills. Conclusions: The prevalence of overall obesity was $26.9 \%$, and the associated factors were older age, genetic predisposition, and diet pills. The prevalence of central obesity was $36.7 \%$, and the associated factors were female gender, older age, and stable marital relationship. There was a good agreement between central and overall obesity. A healthy diet was a protective factor for obesity. The use of diet pills was associated to both types of obesity.

KEY WORDS: ADULT; OBESITY; ABDOMINAL FAT; BODY MASS INDEX; ABDOMINAL CIRCUMFERENCE; RISK FACTORS. 


\section{INTRODUÇÃO}

A obesidade está associada a inúmeras doenças não transmissíveis, contribuindo para o aumento da morbidade e mortalidade por diversas causas. ${ }^{1}$ A obesidade pode ser definida como o grau de armazenamento de gordura em excesso no organismo, associada a riscos para a saúde, devido a sua relação com várias complicações metabólicas. A base da doença é o processo indesejável do balanço energético positivo e perda energética negativa, resultando em ganho de peso. ${ }^{2}$ Vem sendo observado um aumento no número de indivíduos com sobrepeso e obesidade em diversas regiões do país. $^{3}$

Com o avanço da globalização, os alimentos industrializados e o estilo de vida da sociedade moderna têm determinado um padrão alimentar que, aliado ao sedentarismo, não é favorável, em grande parte, à saúde da população. ${ }^{4}$ Desde 1980 a obesidade aumentou consideravelmente. Em 2008, estimou-se que 1,5 bilhões de adultos maiores de 20 anos de idade estavam com peso acima do normal. Destes, aproximadamente 200 milhões de homens e 300 milhões de mulheres eram obesos. ${ }^{5}$

Dados da Vigilância de Fatores de Risco e Proteção para Doenças Crônicas por Inquérito Telefônico (VIGITEL) estimam que em 2011 a obesidade no Brasil variou entre $12,5 \%$ a $21,4 \%$ entre adultos de diferentes regiões brasileiras, com média de $15,8 \%{ }^{6}$

A alta incidência de obesidade tem como causa central o processo denominado transição nutricional, pelas alterações nos padrões de consumo alimentar nas últimas décadas, que consiste na substituição progressiva da escassez alimentar pelo consumo abundante e com maior proporção de alimentos menos saudáveis, fato que está afetando diversas regiões brasileiras, principalmente as áreas com maior urbanização e renda. Segundo a Pesquisa de Orçamentos Familiares (POF) 2008/2009, o consumo de alimentos industrializados teve um percentual duas vezes maior na Região Sudeste $(3,5 \%)$, do que na Região Nordeste $(1,7 \%)$, em detrimento da ingestão de alimentos mais saudáveis e ricos nutricialmente. ${ }^{7}$ Além disso, houve redução importante dos quadros de desnutrição a partir de meados da década de 1970, e com a transição epidemiológica atualmente há no Brasil predomínio de sobrepeso e obesidade em escala populacional. ${ }^{8}$

O objetivo deste estudo foi estimar a prevalência e fatores associados à obesidade geral e à obesidade central entre a população adulta da cidade de Tubarão, Estado de Santa Catarina, Brasil.

\section{MÉTODOS}

\section{Desenho do estudo}

Este estudo é integrante do projeto Estudo da Saúde do Adulto de Tubarão (ESATU), que teve por objetivo avaliar a situação de saúde dos adultos residentes em um município da Região Sul do Brasil. Este é um estudo epidemiológico com delineamento transversal, de base populacional, realizado no período entre novembro de 2011 e junho de 2012, que incluiu participantes de 18 a 59 anos de idade, de ambos os sexos, residentes no Município de Tubarão, Santa Catarina. Foram excluídos do estudo os indivíduos sem condições de deslocamento ao local de coleta dos dados, os que não tinham autonomia para decidir sobre sua participação e as gestantes.

\section{Cálculo do tamanho da amostra}

Considerando que a população adulta em Tubarão era de 62.537 pessoas no censo de 2010, utilizou-se a prevalência estimada de $13,8 \%$ de obesidade, ${ }^{3} \mathrm{com}$ nível de significância de 5\% e margem de erro de $1 \%$, resultando em uma amostra mínima de 174 indivíduos, para um nível de confiança de $95 \%$.

\section{Recrutamento}

Os participantes foram selecionados a partir de amostragem aleatória simples, segundo os números da residência cadastrados em cada uma das 250 microáreas do município, registradas nas 27 unidades de Estratégia de Saúde da Família (ESF). Cada microárea possui um agente comunitário de saúde para realizar visitas domiciliares periódicas, e a taxa de cobertura é estimada em cerca de $90 \%$ da população residente. Os domicílios sorteados foram: 4, 36, 27, 10 e 32. O agente comunitário de saúde de cada micro área se dirigia à primeira casa sorteada (número 4) e convidava o residente a participar do estudo, de acordo com os critérios de inclusão e exclusão. Em caso de mais de um adulto residente no domicílio, que preenchesse os critérios de inclusão, era feita a randomização simples para inclusão no estudo. Caso não houvesse adultos no domicílio ou estes não aceitassem participar do estudo, seguia-se para a casa sorteada subsequente (número 10), até que cada agente comunitário recrutasse de um a dois 
participantes por micro área, com base no tamanho populacional dessa região.

A coleta de dados aconteceu aos sábados, no período matutino, nas dependências ambulatoriais do Hospital Nossa Senhora da Conceição, em consultas pré-agendadas para os participantes sorteados. Solicitou-se aos pacientes que comparecessem em jejum de 12 horas e que evitassem o consumo de bebida alcoólica nas 72 horas anteriores à consulta.

Foram explicados os objetivos do estudo, e solicitou-se a anuência dos pacientes mediante a assinatura do termo de consentimento. Cada participante foi entrevistado por um pesquisador treinado para aplicação do questionário elaborado pelos autores.

\section{Instrumento de coleta de dados}

O instrumento continha perguntas sobre dados sociodemográficos (idade, sexo, cor da pele, situação conjugal, situação de trabalho e escolaridade), dados comportamentais (atividade física, consumo de álcool, tabagismo e passos da alimentação saudável), situação clínica (uso de medicamentos para emagrecer e história familiar de obesidade, indagando se tinha um dos pais ou ambos obesos).

Foram considerados como tabagistas os indivíduos que tinham fumado 100 ou mais cigarros durante a vida. ${ }^{9}$ Entre os adultos que afirmaram consumir álcool atualmente ou tinham consumido no passado, foi aplicado o questionário CAGE - acrônimo em inglês cujos termos significam: diminuir ou cortar o consumo de álcool (Cut Down), sentir-se incomodado por pessoas que o criticam por beber (Annoyed by criticism), ter sentimento de culpa por beber (Guilty) e consumir álcool pela manhã para combater a ressaca ou o nervosismo (Eye-opener). ${ }^{10,11}$

Para aferição da atividade física realizada pelo participante nos últimos sete dias, utilizou-se o Questionário Internacional de Atividade Física, versão curta. ${ }^{12,13}$

Com relação à dieta, foi utilizada uma adaptação dos 10 passos da alimentação saudável propostos pelo Ministério da Saúde (2004). As recomendações foram extraídas do estudo de Vinholes et al., ${ }^{14}$ sendo as recomendações transformadas em perguntas sobre a frequência e quantidade dos alimentos ingeridos com base nas últimas semanas. A partir dessas recomendações, a variável foi utilizada como contínua.

Foi feita a aferição das medidas antropométricas (altura, peso e circunferência abdominal). O peso dos pacientes, trajando roupas leves e sem sapatos, foi medido em quilogramas, com precisão de $0,1 \mathrm{~kg}$. A altura foi medida em centímetros, com precisão de $0,1 \mathrm{~cm}$, com os pacientes tendo os pés unidos, em posição ereta, e olhando para um ponto fixo no horizonte. O peso e a altura foram aferidos em equipamento da marca

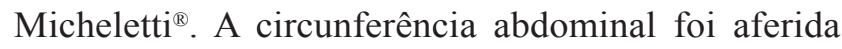
pelo ponto médio central entre a crista ilíaca e a última costela, na altura da cicatriz umbilical, utilizando-se uma trena antropométrica da marca Wiso, modelo T87.

Para a definição de obesidade entre os adultos estudados, foi utilizado o ponto de corte para o índice de massa corporal (IMC) proposto pela Organização Mundial de Saúde (OMS). A OMS propõe que o indivíduo com IMC maior ou igual a $30 \mathrm{~kg} / \mathrm{m}^{2}$ seja considerado obeso em qualquer idade (obesidade geral). ${ }^{15}$ Para a definição de obesidade central dos participantes, utilizou-se a circunferência abdominal (CA), adotando-se a classificação do National Cholesterol Education Program. Segundo tal classificação, $\mathrm{CA} \geq 88 \mathrm{~cm}$ em mulheres e $\geq 102 \mathrm{~cm}$ em homens revela obesidade abdominal. ${ }^{16}$

\section{Análise estatística}

Para o cálculo de tamanho de amostra foi utilizado o programa OpenEpi, versão 2.3.1. ${ }^{17}$ Os dados coletados foram inseridos no programa Epidata, versão 3.1 (EpiData Association, Odense, Denmark), e para a análise estatística foi utilizado o software Statistical Package for Social Sciences (SPSS for Windows v 19; Chicago, IL, USA). Os dados foram descritos com uso da estatística descritiva, frequência e medidas de tendência central e dispersão. Para se verificar a associação entre as variáveis de interesse, foi aplicado teste de quiquadrado de Pearson. Para a comparação entre as médias utilizou-se o teste $t$ de Student. Empregou-se o teste de Kappa para verificar o grau de concordância entre os dois tipos de obesidade. Para controle dos fatores de confusão, foram incluídas na análise as variáveis que apresentaram valor de $\mathrm{p}$ abaixo de 0,20 e/ou selecionadas com base na revisão de literatura, sendo feita análise multivariada pela regressão de Poisson com estimativa robusta de variância. $\mathrm{O}$ intervalo de confiança estabelecido foi de $95 \%$, e erro $\alpha \leq 5 \%$.

\section{Aspectos éticos}

Este estudo foi aprovado pelo Comitê de Ética em Pesquisa da Unisul, sob registro 09.345.4.01.III. O termo de consentimento livre e esclarecido foi assinado por todos os participantes para garantir que a participação 
fosse voluntária. Os dados de identificação dos sujeitos foram mantidos em sigilo pelos pesquisadores.

\section{RESULTADOS}

Dos 419 participantes elegíveis contactados, 376 compareceram para a etapa de coleta de dados e cinco participantes foram excluídos, resultando em amostra final de 371 indivíduos. A taxa de não resposta foi, portanto, de $11,5 \%$.

Foram estudados 371 pacientes adultos, com média de idade de 40,5 (desvio padrão [DP] =12,3 anos), dos quais $63,9 \%$ eram mulheres. Com relação à obesidade, $26,9 \%$ dos participantes foram considerados obesos pelo ponto de corte do IMC, e $36,7 \%$ pelo da CA. Ao se verificar o grau de concordância entre a obesidade geral e central obteve-se um valor de Kappa de 0,7, considerado um bom grau de concordância. Comparando-se os resultados, 43,5\% das mulheres apresentaram circunferência abdominal aumentada, enquanto $24,6 \%$ dos homens apresentaram tal aumento, com um valor de $\mathrm{p}<0,001$.

A Tabela 1 apresenta o perfil e as características sociodemográficas da população em estudo e sua distribuição entre os obesos. A circunferência abdominal variou entre 61,0 e $180,0 \mathrm{~cm}$, com média de $89,0 \mathrm{~cm}(\mathrm{DP}=15,0)$. A altura variou entre $141,0 \mathrm{e}$ $193,0 \mathrm{~cm}$, com média de $163,0 \mathrm{~cm}(\mathrm{DP}=9,16)$, enquanto que o peso variou entre $40,7 \mathrm{~kg}$ e $129,7 \mathrm{~kg}$, com média de 73,4 kg $(\mathrm{DP}=17,0)$, entre os participantes do estudo. A amostra apresentou um predomínio de mulheres, etnia branca, na faixa etária entre 40-49 anos de idade, que tinha relacionamento estável e atividade laboral.

A obesidade geral apresentou associação com idade mais avançada, menor escolaridade e relacionamento estável. A obesidade central apresentou associação com as mesmas variáveis, além da associação positiva com o sexo feminino (Tabela 1).

A Tabela 2 apresenta os passos adaptados da alimentação saudável dos adultos participantes do estudo. Apenas 38,0\% dos participantes foram considerados adeptos de uma dieta saudável, seguindo cinco ou mais recomendações dos passos da alimentação saudável.

$\mathrm{O}$ passo que apresentou maior adesão foi o de número 7 , sendo que $85,7 \%$ dos indivíduos informaram evitar o consumo diário de álcool e refrigerante. O consumo diário de frutas e verduras foi o passo com menos adeptos, pois $96,2 \%$ dos entrevistados não fazem o consumo diário destes alimentos. Considerando a variável de forma contínua, houve diferença estatisticamente significativa entre a média de recomendações seguidas e a obesidade. Os participantes não obesos apresentaram uma média de 4,3 passos da alimentação saudável contra 3,7 dos participantes com obesidade central $(\mathrm{p}<0,001)$ e 3,7 com obesidade geral $(p=0,002)$ (Tabela 2).

Tabela 1. Características sociodemográficas da população em estudo e sua distribuição entre os obesos $(n=371)$.

\begin{tabular}{|c|c|c|c|c|c|}
\hline Características & $\begin{array}{c}\text { Número total } \\
\text { n (\%) }\end{array}$ & $\begin{array}{l}\text { Obesidade geral } \\
\qquad \begin{array}{c}(n=100) \\
n(\%)\end{array}\end{array}$ & $p$ & $\begin{array}{l}\text { Obesidade central } \\
\qquad \begin{array}{c}(n=136) \\
n(\%)\end{array}\end{array}$ & $p$ \\
\hline $\begin{array}{l}\text { Sexo } \\
\text { Masculino } \\
\text { Feminino }\end{array}$ & $\begin{array}{l}134(36,1) \\
237(63,9)\end{array}$ & $\begin{array}{l}31(31,0) \\
69(69,0)\end{array}$ & 0,212 & $\begin{array}{c}33(24,3) \\
103(75,7)\end{array}$ & $<0,001$ \\
\hline $\begin{array}{l}\text { Idade (em anos) } \\
\qquad \begin{array}{l}18-39 \\
40-49 \\
\geq 50\end{array}\end{array}$ & $\begin{array}{l}151(40,7) \\
111(29,9) \\
109(29,4)\end{array}$ & $\begin{array}{l}31(31,0) \\
26(26,0) \\
43(43,0)\end{array}$ & 0,002 & $\begin{array}{l}34(25,0) \\
38(27,9) \\
64(47,1)\end{array}$ & $<0,001$ \\
\hline $\begin{array}{l}\text { Etnia }(\mathrm{n}=370)^{*} \\
\text { Brancos } \\
\text { Não brancos }\end{array}$ & $\begin{array}{c}320(86,5) \\
50(13,5)\end{array}$ & $\begin{array}{l}89(89,0) \\
11(11,0)\end{array}$ & 0,389 & $\begin{array}{c}120(88,2) \\
16(11,8)\end{array}$ & 0,453 \\
\hline $\begin{array}{l}\text { Escolaridade } \\
\begin{array}{c}\text { 0-8 anos } \\
>8 \text { anos }\end{array}\end{array}$ & $\begin{array}{l}183(49,3) \\
188(50,7)\end{array}$ & $\begin{array}{l}59(59,0) \\
41(41,0)\end{array}$ & 0,024 & $\begin{array}{l}85(62,5) \\
51(37,5)\end{array}$ & $<0,001$ \\
\hline $\begin{array}{l}\text { Situação conjugal } \\
\text { Sem companheiro } \\
\text { Com companheiro }\end{array}$ & $\begin{array}{l}115(31,0) \\
256(69,0)\end{array}$ & $\begin{array}{l}22(22,0) \\
78(78,0)\end{array}$ & 0,023 & $\begin{array}{c}26(19,1) \\
110(80,9)\end{array}$ & $<0,001$ \\
\hline $\begin{array}{l}\text { Trabalha } \\
\text { Sim } \\
\text { Não }\end{array}$ & $\begin{array}{l}244(65,8) \\
127(34,2)\end{array}$ & $\begin{array}{l}58(58,0) \\
42(42,0)\end{array}$ & 0,055 & $\begin{array}{l}82(60,3) \\
54(39,7)\end{array}$ & 0,091 \\
\hline
\end{tabular}

Obesidade geral aferida pelo índice de massa corporal.

Obesidade central aferida pela circunferência abdominal.

* 71 sem informação sobre a etnia. 
Tabela 2. Frequência de cada uma das recomendações de passos da alimentação saudável do Ministério da Saúde - adaptado*. $(n=371)$.

\section{Recomendações de Alimentação Saudável - adaptado}

Passo 1: Consumo diário de frutas e verduras

Passo 2: Consumo de feijão pelo menos $4 \mathrm{x} / \mathrm{semana}$

Passo 3: Consumo máximo de 1x/semana de alimentos gordurosos

Passo 4: Não adição de sal aos alimentos já servidos no prato

Passo 5: Consumo de pelo menos três refeições e um lanche por dia

Passo 6: Consumo máximo de 2x/semana de alimentos ricos em açúcar

Passo 7: Evitar o consumo diário de álcool e refrigerantes

Passo 8: Manter o peso dentro dos limites saudáveis, IMC: $18,5-24,9 \mathrm{~kg} / \mathrm{m}^{2}$

Passo 9: Prática diária de atividade física por, no mínimo, 30 minutos

Passo 10: Ingerir, no mínimo, 2 litros de água por dia

* Com base no estudo de Vinholes et al. ${ }^{1+}$

$\mathbf{n}(\%)$
$14(3,8)$
$195(52,6)$
$70(18,9)$
$314(84,6)$
$208(56,1)$
$130(35,0)$
$318(85,7)$
$146(39,4)$
$146(39,4)$
$122(32,9)$

$122(32,9)$
A Tabela 3 apresenta os hábitos e estilo de vida dos participantes do estudo e sua associação com a presença de obesidade geral ou central.

A Tabela 4 mostra os resultados da análise multivariada, com os fatores associados de forma independente à obesidade geral pelo IMC e central pela CA. Com relação à obesidade geral, houve associação independente com faixa etária maior, histórico familiar de obesidade e uso de medicamentos para emagrecimento. Com relação à obesidade central, houve associação independente com sexo feminino, maior faixa etária, ter união estável, história familiar de obesidade e uso de medicamentos. A dieta saudável foi considerada fator de proteção para obesidade geral e central.

Tabela 3. Hábitos e estilo de vida, e sua distribuição entre os participantes com obesidade. $(n=371)$.

\begin{tabular}{|c|c|c|c|c|c|}
\hline Hábitos e história & $\begin{array}{c}\text { Número total } \\
\text { n (\%) }\end{array}$ & $\begin{array}{l}\text { Obesidade geral } \\
\qquad \begin{array}{c}(n=100) \\
n(\%)\end{array}\end{array}$ & $p$ & $\begin{array}{l}\text { Obesidade central } \\
\qquad(n=136) \\
n(\%)\end{array}$ & $p$ \\
\hline $\begin{array}{l}\text { Atividade Física } \\
\text { Sim } \\
\text { Não }\end{array}$ & $\begin{array}{l}146(39,4) \\
225(60,6)\end{array}$ & $\begin{array}{l}36(36,0) \\
64(64,0)\end{array}$ & 0,422 & $\begin{array}{l}44(32,4) \\
92(67,6)\end{array}$ & 0,036 \\
\hline $\begin{array}{l}\text { Pais obesos } \\
\text { Sim } \\
\text { Não }\end{array}$ & $\begin{array}{l}138(37,2) \\
233(62,8)\end{array}$ & $\begin{array}{l}53(53,0) \\
47(47,0)\end{array}$ & $<0,001$ & $\begin{array}{l}61(44,9) \\
75(55,1)\end{array}$ & 0,020 \\
\hline $\begin{array}{l}\text { Moderadores de apetite } \\
\text { Sim } \\
\text { Não }\end{array}$ & $\begin{array}{c}18(4,9) \\
353(95,1)\end{array}$ & $\begin{array}{l}12(12,0) \\
88(88,0)\end{array}$ & $<0,001$ & $\begin{array}{c}13(9,6) \\
123(90,4)\end{array}$ & 0,002 \\
\hline $\begin{array}{l}\text { Tabagismo } \\
\text { Fumante } \\
\text { Ex-fumante } \\
\text { Nunca fumou }\end{array}$ & $\begin{array}{c}40(10,8) \\
76(20,5) \\
255(68,7)\end{array}$ & $\begin{array}{l}10(10,0) \\
23(23,0) \\
67(67,0)\end{array}$ & 0,756 & $\begin{array}{c}12(8,8) \\
37(27,2) \\
87(64,0)\end{array}$ & 0,045 \\
\hline $\begin{array}{l}\text { Alcoolista } \\
\text { Sim } \\
\text { Não }\end{array}$ & $\begin{array}{c}31(8,4) \\
340(91,6)\end{array}$ & $\begin{array}{c}7(7,0) \\
93(93,0)\end{array}$ & 0,566 & $\begin{array}{c}11(8,1) \\
125(91,9)\end{array}$ & 0,887 \\
\hline
\end{tabular}

Obesidade geral aferida pelo índice de massa corporal. Obesidade central aferida pela circunferência abdominal.

Tabela 4. Análise multivariada, usando o modelo de regressão de Poisson, dos fatores associados à obesidade.

\begin{tabular}{lcccc}
\multicolumn{1}{c}{ Fatores } & \multicolumn{2}{c}{ Obesidade geral } & \multicolumn{2}{c}{ Obesidade central } \\
\cline { 2 - 5 } \multicolumn{1}{c}{ RP ajustada* } & $\mathbf{p}$ & $\mathbf{R}$ ajustada* & $\boldsymbol{p}$ \\
\hline Sexo feminino & $1,17(0,80-1,72)$ & 0,421 & $1,86(1,35-2,57)$ & $<\mathbf{0 , 0 0 1}$ \\
Idade & $1,03(1,01-1,05)$ & $<\mathbf{0 , 0 0 1}$ & $1,04(1,02-1,05)$ & $<\mathbf{0 , 0 0 1}$ \\
Menor escolaridade & $1,10(0,76-1,59)$ & 0,625 & $1,19(0,90-1,59)$ & 0,222 \\
União estável & $1,20(0,80-1,80)$ & 0,384 & $1,40(0,99-1,97)$ & $\mathbf{0 , 0 5 5}$ \\
Trabalho & $0,74(0,54-1,03)$ & 0,075 & $0,93(0,73-1,19)$ & 0,554 \\
Atividade física & $1,05(0,72-1,55)$ & 0,795 & $0,95(0,71-1,26)$ & 0,710 \\
Dieta saudável & $0,80(0,70-0,92)$ & $\mathbf{0 , 0 0 1}$ & $0,79(0,72-0,87)$ & $<\mathbf{0 , 0 0 1}$ \\
Pais obesos & $1,77(1,27-2,46)$ & $\mathbf{0 , 0 0 1}$ & $1,32(1,04-1,68)$ & $\mathbf{0 , 0 2 4}$ \\
Moderadores de apetite & $1,99(1,33-2,99)$ & $\mathbf{0 , 0 0 1}$ & $1,64(1,11-2,44)$ & $\mathbf{0 , 0 1 4}$ \\
\hline Tabagismo & $0,80(0,56-1,14)$ & 0,222 & $0,96(0,74-1,23)$ & 0,720 \\
\hline
\end{tabular}

* Ajustada para todas as variáveis independentes: sexo, idade (contínua), escolaridade (contínua), união estável, trabalho, atividade física, dieta (contínua), história familiar de obesidade, uso de medicamentos moderadores de apetite e tabagismo. Obesidade geral: aferida pelo índice de massa corporal. Obesidade central: aferida pela circunferência abdominal.

RP: razão de prevalência. 


\section{DISCUSSÃO}

No presente estudo, a prevalência de obesidade foi de $26,9 \%$ pelo IMC (obesidade geral) e de $36,7 \%$ pela classificação da CA (obesidade abdominal). Esta prevalência é maior quando comparada com as de outras regiões brasileiras. O estudo de Gigante et al., que avaliou a variação temporal da prevalência do excesso de peso e obesidade em adultos no Brasil, encontrou valores crescentes de obesidade geral na população no decorrer dos anos, sendo $11,4 \% \mathrm{em}$ 2006, 12,7\% em 2007, 13,2\% em 2008 e 13,8\% em 2009. ${ }^{3}$ Estudo realizado no Acre, no período entre 2007 e 2008, encontrou prevalência de 15,9\% de obesidade geral na população estudada. ${ }^{18}$ No entanto, Holanda et al. ${ }^{19} \mathrm{em}$ estudo realizado na região urbana de Teresina, Piauí, encontraram apenas $7,7 \%$ de obesidade geral.

A obesidade central está associada a distúrbios metabólicos e doenças cardiovasculares. A obesidade abdominal pode elevar em até dez vezes o risco para o desenvolvimento de diabetes tipo 2 , além de também constituir-se em fator de risco para hipertensão arterial em adultos com idade entre 20 e 45 anos. ${ }^{4,19}$ Isso revela a preocupação com os dados encontrados no presente estudo, já que mais de um terço da amostra apresentou obesidade abdominal. A obesidade geral, apesar de ser amplamente utilizada em estudos epidemiológicos e na prática clínica para o diagnóstico da obesidade, por ser um método de baixo custo e não invasivo, ela possui alguns fatores limitantes com o emprego do IMC, como a falta de ajustes com relação ao peso ósseo e muscular, podendo por vezes não ser um bom indicar de obesidade. Contudo, os pontos de corte definidos pela OMS geralmente são estabelecidos pela curva de mortalidade associada a maior IMC. ${ }^{4}$

Parece haver consenso de que os principais fatores associados ao ganho excessivo de peso envolve a interação de fatores sociais, econômicos, comportamentais, culturais, fisiológicos, metabólicos e genéticos. ${ }^{16}$ Os fatores comportamentais ganham destaque no padrão de consumo alimentar mais comum atualmente, com base na excessiva ingestão de alimentos de alta densidade energética, ricos em açúcares, gordura saturada, sódio e conservantes, porém pobres em fibras e nutrientes. Além disso, observa-se um percentual excessivo e crescente de sedentarismo na sociedade moderna. ${ }^{20}$

As mulheres apresentaram maior prevalência de obesidade central, sendo o sexo feminino um fator associado de forma independente ao aumento de CA, fato que também se observou nos estudos de Holanda et al. ${ }^{19}$ e Foulds et al. ${ }^{21}$ Isso pode ser atribuído ao fato de a mulher armazenar maior quantidade de gordura no período pós-prandial, já que o estrógeno tem o efeito de reduzir a oxidação do ácido que atuaria na quebra da gordura. ${ }^{22} \mathrm{Na}$ verdade, as mulheres apresentam maior adiposidade que os homens, principalmente no que se refere a gordura visceral e central, sendo que a percentagem de gordura encontrada em mulheres com IMC considerado normal corresponde ao encontrado em homens com IMC superior a $30 .{ }^{23}$

Além disso, as mulheres metabolizam maior quantidade de gordura e menos aminoácidos e carboidratos durante as atividades físicas, sendo esta a causa atribuível aos maiores níveis de 17-alfa-estradiol encontrados em mulheres do que em homens. ${ }^{24}$ Também há fatores fisiológicos associados ao excesso de peso em mulheres. $\mathrm{O}$ ciclo reprodutivo determina que quanto mais precoce a idade da menarca, maior a chance de obesidade na vida adulta, assim como a paridade, o maior número de gestações e a menopausa, quando ocorre perda de massa óssea e aumento do acúmulo de gordura abdominal. ${ }^{25}$

Os resultados deste estudo são consistentes com a literatura no que se refere ao efeito da idade na obesidade, classificada tanto pelo IMC quanto pela CA. ${ }^{18,} 26$ No modelo final, para determinantes de obesidade, a idade mais avançada apresentou razão de prevalência de 1,03 vezes, tanto na obesidade geral, quanto na central. Tal fato é semelhante ao apresentado em outros estudos. ${ }^{18,26} \mathrm{O}$ envelhecimento justifica o ganho de peso em razão de fatores como declínio na taxa de metabolismo basal e perda de massa muscular, com aumento da proporção de gordura corpórea, aumento no consumo alimentar e diminuição da atividade física. ${ }^{19,27}$

Houve uma associação estatisticamente significativa, na análise bivariada, entre menor escolaridade e obesidade central, como também foi observado no estudo de Faeh et al., ${ }^{28}$ que verificaram que, quanto maior a escolaridade, menor a chance do excesso de peso, principalmente em mulheres adultas. Acredita-se que a população de baixa escolaridade apresenta uma probabilidade maior de aumento de peso devido à falta de informação, piores condições de vida, falta de orientação para redução de peso, menor adesão a um estilo de vida saudável e consumo de alimentos de baixo custo, porém com elevada densidade energética. ${ }^{29}$ Entretanto, após o ajuste das variáveis de confusão, não houve associação entre obesidade e grau de escolaridade. Tzotzas et al..$^{30}$ também não encontraram associação entre grau de escolaridade e obesidade. 
Apenas $38 \%$ dos participantes deste estudo aderiram a uma dieta saudável, que foi fator protetor contra a obesidade abdominal. A evolução dos padrões de consumo alimentar ocorrido nas últimas três décadas no Brasil e no mundo evidenciou declínio do consumo de alimentos básicos e tradicionais, como feijão e arroz, acompanhado por aumento de até $400 \%$ do consumo de produtos industrializados, persistência do consumo excessivo de açúcar e gorduras e consumo insuficiente de frutas e verduras na dieta, o que contribui para o aumento das doenças e agravos não transmissíveis. ${ }^{31}$ Este grupo de doenças representa a principal causa de morte e mais de $70 \%$ dos gastos de assistência em saúde no Brasil, com tendência crescente nas áreas em desenvolvimento. ${ }^{5}$ Há estudos que apontam a alimentação não saudável e o sedentarismo como os principais determinantes do aumento expressivo na prevalência das doenças e agravos não transmissíveis. A OMS indica que cerca de $80 \%$ das doenças coronarianas, $90 \%$ do diabetes tipo 2 e $30 \%$ das neoplasias poderiam ser evitados por meio de práticas alimentares saudáveis, maior nível de atividade física e menor utilização do tabaco e seus derivados. ${ }^{32}$

No presente estudo, a prática de atividade física apresentou uma associação inversa à obesidade central na análise univariada; porém, após o ajuste das variáveis de confusão, perdeu a significância estatística. Foi encontrado resultado similar no estudo de Ekelund et al., que afirmaram que a prática de atividade física ao longo do tempo pode induzir a mudanças na composição e redistribuição da gordura corporal, sem reduzir o peso corporal. ${ }^{33}$ Há estudos que sugerem que o exercício físico de intensidade moderada esteja associado a um aumento da lipólise no tecido celular subcutâneo abdominal em relação a outros locais do corpo. ${ }^{34}$

Em relação à predisposição genética (histórico de pais obesos), o presente estudo constatou que $53 \%$ dos participantes apresentavam obesidade geral e 44,9\% apresentavam obesidade abdominal. Essa relação permaneceu significativa após o ajuste das variáveis de confusão, mostrando que a história familiar de obesidade é um fator associado de forma independente à presença de obesidade geral e central. Resultados similares foram encontrados no estudo de Gigante et al., ${ }^{35}$ que mostrou que a obesidade de um dos pais ocasionou chance $50 \%$ maior de os filhos serem obesos, comparativamente àqueles que responderam que nenhum dos pais era obeso. A transmissão familiar de obesidade está relacionada aos genes que intervêm na manutenção de peso e gordura estáveis ao longo do tempo, pela sua participação no controle de vias eferentes (leptina, nutrientes, sinais nervosos, entre outros), de mecanismos centrais (neurotransmissores hipotalâmicos) e de vias aferentes (insulina, catecolaminas, sistema nervoso autônomo). Desta forma, o balanço energético, do qual participam a energia ingerida e a energia gasta, parece depender de cerca de $40 \%$ da herança genética, podendo afetar ambas as partes da equação energética (apetite e gasto). ${ }^{36}$

O uso de medicamentos para emagrecer foi considerado um fator associado de forma independente à ocorrência de obesidade geral e central. A efetividade em longo prazo dos inibidores de apetite é, na melhor das hipóteses, questionável. Embora causem perda de peso nas primeiras semanas de tratamento, a redução de peso atribuível aos anorexígenos é, em geral, modesta e a recuperação parcial de peso ocorre quando eles são usados por período superior a um ano. ${ }^{37}$ Contudo, por se tratar de um estudo transversal, esse achado sofre do fenômeno de causalidade reversa: não é possível determinar se pelo fato de os pacientes serem obesos utilizavam medicamentos para combater esta doença, ou se já utilizaram esses medicamentos no passado e não houve efetividade.

$\mathrm{O}$ tabagismo mostrou-se um fator associado ao ganho de peso abdominal, fato também constatado no estudo de Castanheira et al., ${ }^{38} \mathrm{em}$ que foi observada maior média de perímetro abdominal entre os homens e mulheres ex-fumantes. O mecanismo que poderia explicar essa associação é o aumento da taxa metabólica basal provocada pelos componentes do fumo, que poderia implicar aumento no consumo alimentar com a interrupção do vício. ${ }^{30}$ Entretanto, após o ajuste das variáveis de confusão, esta variável perdeu a significância estatística.

Entre as limitações do presente estudo destaca-se o delineamento, uma vez que em estudos transversais não é possível determinar causa e efeito. Além disso, os métodos utilizados para a definição de obesidade podem sofrer alterações decorrentes da composição corporal individual, principalmente no que se refere ao IMC. O deslocamento até o hospital para a entrevista e coleta de dados pode ter dificultado o acesso de pessoas com obesidade severa ou pode ter estimulado a participação de indivíduos com outros problemas de saúde em busca de tratamento, além de uma taxa de não resposta de $11,5 \%$. Por outro lado, a amostra foi maior que a mínima calculada e o método adotado, com a randomização dos domicílios, permitiu uma aferição mais adequada das variáveis do estudo, por equipe tecnicamente treinada para essa função, em 
ambiente seguro e controlado. Ressalte-se que, por se tratar de estudo populacional, estes dados refletem a realidade local e podem servir de subsídios para a implementação de políticas públicas de prevenção e tratamento da obesidade na população do município de Tubarão e região. Além disso, este estudo permite a comparação dos achados com outras realidades populacionais com características similares.

Com base nestes resultados, conclui-se que houve boa concordância entre prevalência de obesidade geral, aferida pelo IMC, e obesidade central, aferida pela CA. Idade mais avançada, predisposição genética e uso de medicamentos para emagrecer foram fatores associados de forma independente aos dois tipos de obesidade. Com relação à obesidade central, somaram-se como fatores associados de forma independente o sexo feminino e manter união estável. A dieta saudável foi fator de proteção para obesidade geral e central. A elevada prevalência de obesidade encontrada nesta população evidencia a necessidade de medidas públicas para a prevenção desta comorbidade, e intervenção multidisciplinar para implementar mudanças comportamentais em longo prazo, com ênfase em nutrição adequada e exercício físico, podendo-se evitar doenças decorrentes da obesidade ou a ela associadas.

\section{REFÊRENCIAS}

1. Lessa I. O adulto brasileiro e as doenças da modernidade: epidemiologia das doenças crônicas não transmissíveis. São Paulo: Hucitec; 1998. $284 \mathrm{p}$

2. Wanderley EN, Ferreira VA. Obesidade: uma perspectiva plural. Cienc Saude Colet. 2010;15(1):185-94.

3. Gigante DP, França GV, Sardinha LMV, Iser BPM, Meléndez GV. Temporal variation in the prevalence of weight and obesity excess in adults: Brazil, 2006 to 2009. Rev Bras Epidemol. 2011;14(1):157-65.

4. James WP. The epidemiology of obesity: the size of the problem. J Intern Med. 2008; 263(4):336-52.

5. Brasil. Ministério da Saúde [Internet]. Brasília (DF); 2014 [updated 2014; cited 2014 Jul. 20]. Available from: http://portal.saude.gov.br/ saude/visualizar_texto.cfm?idtxt $=25341$

6. Brasil. Ministério da Saúde. Secretaria de Vigilância em Saúde. Vigitel Brasil 2011: Vigilância de Fatores de Risco e Proteção para Doenças Crônicas por Inquérito Telefônico. Brasília (DF): Ministério da Saúde; 2012.

7. Brasil. Ministério do Planejamento, Orçamento e Gestão. Instituto Brasileiro de Geografia e Estatística - IBGE. Pesquisa de orçamentos familiares 2008-2009. Despesas, rendimentos e condições de vida, 2008-2009 [Internet]. Rio de Janeiro; 2010. [updated 2010; cited 2014 Jul 20]. Available from: http://www.ibge.gov.br/home/estatistica/populacao/condicaodevida/pof/2008_2009/POFpublicacao.pdf

8. Batista Filho M, Rissin A. A transição nutricional no Brasil: tendências regionais e temporais. Cad Saúde Pública 2003;19(Suppl 1): S181-S91.

9. Reichert J, Araújo AJ, Gonçalves C, Godoy I, Chatkin JM, Sales M. Diretrizes para cessação do tabagismo. J Bras Pneumol. 2008;34(10): 845-80.

10. Mayfield D, McLeod G, Hall P. The CAGE questionnaire: Validation of a new alcoholism instrument. Am J Psychiatry. 1974;131(10):1121-3.

11. Castells MA, Furlanetto LM. Validity of the CAGE questionnaire for screening alcohol-dependent inpatients on hospital wards. Rev Bras Psiquiatr 2005;27(1):54-7.

12. Guidelines for Data Processing and Analysis of the International Physical Activity Questionnaire (IPAQ): Short and Long Forms. [Internet] 2005; [cited 2014 Jul 20]. Available from: https://sites.google.com/site/theipaq/

13. Pate RR, Pratt M, Blair SN, Haskell WL, Macera CA, Bouchard C, Ettinger W, Heath GW, King AC, Kriska A, Leon AS, Marcus BH, Morris J, Paffenbarger Jr RS, Patrick K, Pollock ML, Rippe JM, Sallis J, Wilmore JH. Physical activity and public health. A recommendation from the centers for disease control and prevention and the American college sports medicine. JAMA. 1995;237(5):402-7.

14. Vinholes DB, Assunção MCF, Neutzling MB. Frequência de hábitos saudáveis de alimentação medidos a partir dos 10 Passos da Alimentação Saudável do Ministério da Saúde. Pelotas, Rio Grande do Sul, Brasil. Cad Saúde Pública. 2009;25(4):791-9.

15. World Health Organization - WHO. [World health report] [Internet]. [cited 2014 Jul 22]. Available from: http://www.who.int/whr/en/

16. National Obesity Education Initiative of the National Heart, Lung, and Blood Institute. Clinical Guidelines on the Identification, Evaluation, and Treatment of Overweight and Obesity in Adults: the evidence report. Obes Res. 1998 Sept; 6 Suppl 2:51S-209S.

17. Dean AG, Sullivan KM, Soe MM. OpenEpi: Open Source Epidemiologic Statistics for Public Health, Version 2.3.1 [Internet]. [cited 2014 Jul 15]. Available from: www.OpenEpi.com.

18. Lino MRZ, Muniz PT, Siqueira KS. Prevalência e fatores associados ao excesso de peso em adultos: inquérito populacional em Rio Branco, Acre, Brasil, 2007-2008 Cad Saúde Pública. 2011;27(4):797-810.

19. Holanda LGM, Martins MCC, Souza Filho MD, Carvalho CMRG, Asis RC, Leal LMM, Costa EM. Excesso de peso e adiposidade central em adultos de Teresina-PI. Rev Assoc Med Bras. 2011;57(1):50-5.

20. Brasil. Ministério da Saúde. Secretaria de Atenção à Saúde. Departamento de Atenção Básica. Cadernos de Atenção Básica no. 12: Obesidade. Brasília (DF); 2006. [cited 2014 Jul 20]. Available from: http://189.28.128.100/nutricao/docs/geral/doc_obesidade.pdf 
21. Foulds HJ, Bredin SS, Warburton DE. The prevalence of overweight and obesity in British Columbian Aboriginal adults. Obes Rev. 2011; 12(5):e4-e11.

22. O'Sullivan AJ. Does oestrogen allow women to store fat more efficiently? A biological advantage for fertility and gestation. Obes Rev. 2009;10(2):168-77.

23. Power ML, Schulkin J. Sex differences in fat storage, fat metabolism, and the health risks from obesity: possible evolutionary origins. Br J Nutr 2008;99(5):931-40.

24. Tarnopolsky MA. Sex differences in exercise metabolism and the role of 17-beta estradiol. Med Sci Sports Exerc. 2008;40(4):648-54.

25. Teichmann L, Olinto MTA, Costa JSD, Ziegler D. Fatores de risco associado ao sobrepeso e obesidade em mulheres de São Leopoldo, RS. Rev Bras Epidemol. 2006;9(3):360-73.

26. Oliveira LPM, Assis AM, Silva MCM, Santana MLP, Santos NS, Pinheiro SMC, Barreto ML, Souza CO. Fatores associados a excesso de peso e concentração de gordura abdominal em adultos na cidade de Salvador, Bahia, Brasil. Cad Saúde Pública. $2009 ; 25(3): 570-82$.

27. Steeves JA, Bassett DR, Thompson DL, Fitzhugh EC. Relationships of occupational and non-occupational physical activity to abdominal obesity. Int J Obes. 2012;36(1):100-6.

28. Faeh D, Braun J, Boop M. Prevalence of obesity in Switzerland 1992-2007: the impact of education, income and occupational class Obes Rev. 2011;12(3):151-66.

29. Gutierrez- Fisac JL, Regidor E, Banegas JRB, Artalejo FR. The size of obesity differences associated with educational level in Spain, 1987-1995/97. J Epidemiol Community Health. 2002;56(6):457-60.

30. Tzotzas T, Vlahavas G, Papadopoulou SK, Kapantais E, Kaklamanou D, Hassapidou M. Marital status and educational level associated to obesity in Greek adults: data from the National Epidemiological Survey. BMC Public Health. 2010;10:732. doi: 10.1186/14712458-10-732.

31. World Health Organization. Food and Agriculture Organization [WHO/FAO]. Diet, nutrition and prevention of chronic diseases. Geneva; 2003. [WHO - Technical Report Series, 916]. Acesso em: [2013 Maio 5]. Disponível em: http://www.who.int/dietphysicalactivity/ publications/trs916/download/en/index.html

32. Organização Pan-Americana da Saúde (OPAS). Doenças crônico-degenerativas e obesidade: estratégia mundial sobre alimentação saudável, atividade física e saúde. Brasília: Ministério da Saúde; 2003. Acesso em: [2013 Maio 5]. Disponível em: http://portal.saude. gov.br/portal/arquivos/mp3/diretrizes_recomendacoes_dent.pdf

33. Ekelund U, Besson H, Luan J, May AM, Sharp SJ, Brage S, Travier N, Agudo A, Slimani N, Rinaldi S, Jenab M, Norat T, Mouw T, Rohrmann S, Kaaks R, Bergmann MM, Boeing H, Clavel-Chapelon F, Boutron-Ruault MC, Overvad K, Jakobsen MU, Johnsen NF, Halkjaer J, Gonzalez CA, Rodriguez L, Sanchez MJ, Arriola L, Barricarte A, Navarro C, Key TJ, Spencer EA, Orfanos P, Naska A, Trichopoulou A, Manjer J, Lund E, Palli D, Pala V, Vineis P, Mattiello A, Tumino R, Bueno-de-Mesquita HB, van den Berg SW, Odysseos AD, Riboli E, Wareham NJ, Peeters PH. Physical activity and gain in abdominal adiposity and body weight: prospective cohort study in 288,498 men and women. Am J Clin Nutr. 2011;93(4):826-35.

34. Arner P, Kriegholm E, Engfeldt P, Bolinder J. Adrenergic regulation of lipolysis in situ at rest and during exercise. J Clin Invest. 1990;85(3):893-8.

35. Gigante DP, Barros FC, Post CLA, Olinto MTA. Prevalence and risk factors of obesity in adults. Rev Saúde Pública. 1997;31(3): 236-46.

36. Marques-Lopes I, Marti A, Moreno-Aliaga MJ, Martinez A. Genetics of obesity. Rev Nutr. 2004;17(3):327-38.

37. Zanella MT, Ribeiro Filho FF. Emerging drugs for obesity therapy. Arq Bras Endocrinol Metabol. 2009;53(2):271-80.

38. Castanheira M, Olinto MTA, Gigante DP. Socio-demographic and lifestyle factors associated with abdominal fat distribution in adults: a population-based survey in Southern Brazil. Cad Saúde Pública. 2003;19(1):S55-S65. 